\title{
Composition Sensitivity for the Cosmic Ray Anisotropy with SWGO
}

\section{Andrew M. Taylor, ${ }^{a}, *$ Gwenael Giacinti, ${ }^{b}$ Paolo Desiati, ${ }^{c}$ Juan Carlos Diaz Velez, ${ }^{c}$ Andrea Chiavassa, ${ }^{d}$ Guiseppe Di Sciascio, ${ }^{e}$ Juan Carlo Arteaga Velazquez ${ }^{f}$ and Samridha Kunwar ${ }^{b}$}

${ }^{a}$ DESY, Platanenallee 6, 15738 Zeuthen, Germany

${ }^{b}$ MPIK, Saupfercheckweg 1, 69117 Heidelberg, Germany

${ }^{c}$ WIPAC, University of Wisconsin - Madison, 222 W. Washington Ave. Madison, WI 53703, U.S.A.

${ }^{d}$ Dipartimento di Fisica, Università degli Studi di Torino, Torino, Italy

${ }^{e}$ Istituto Nazionale di Fisica Nucleare, Sezione di Roma Tor Vergata, via della Ricerca Scientifica 1, 00133 Roma, Italy

${ }^{f}$ Institute of Physics and Mathematics, Universidad Michoacana de San Nicol as de Hidalgo, Morelia, Mexico

E-mail: andrew.taylor@desy.de, Gwenael.Giacinti@mpi-hd.mpg.de

A number of cosmic-ray observatories have measured a change in both phase and amplitude of the dipole component in the distribution of cosmic-ray arrival directions above a primary energy of $100 \mathrm{TeV}$. We focus on probing the cosmic-ray dipole and multipole evolution in the energy region of mutli $\mathrm{TeV}$ to beyond $\mathrm{PeV}$ with a future large-area gamma-ray observatory, such as the Southern Wide-field Gamma-ray Observatory (SWGO). The ability to discriminate between different mass groups is essential to understand the origin of this evolution. Through a consideration of the energy and mass resolution for cosmic-ray detection by such an observatory, we estimate its separation power for decomposing the full-particle anisotropy into mass groups. In particular, we explore the feasibility of probing the dipole evolution with rigidity with SWGO. In this way, we demonstrate the great potential that this instrument offers for providing a deeper understanding of the origin of the cosmic-ray anisotropy.

$37^{\text {th }}$ International Cosmic Ray Conference (ICRC 2021)

July 12th - 23rd, 2021

Online - Berlin, Germany

\footnotetext{
*Presenter
} 


\section{Introduction}

One of the most direct probes into the origin of cosmic rays comes from studies of their arriving anisotropy. Although these particles arrive largely isotropically, the level of their underlying dipole and multipoles have in recent years started to be probed [1]. In particular, a large phase swing of the dipole direction, and a corresponding dip in the overall dipole amplitude, has been observed in the preceding two energy decades below the knee. The origin of this feature, however, remains unclear.

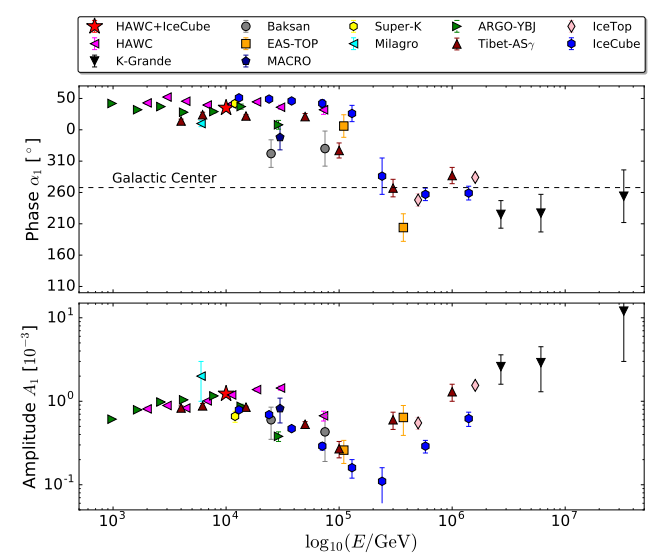

Figure 1: The evolution in the phase (top-panel) and amplitude (bottom-panel) of the cosmic ray dipole, which exhibits a strong swing in the two energy decades below the knee energy scale. This plot has been taken from [1].

We here explore the role that the future detector SWGO, located in the southern hemisphere on Earth, can play in providing a better understanding for the origin of this feature. Specifically, we focus on this instruments ability to separate out the arriving cosmic rays into several major mass groups.

\section{Dipole Probe Energy Range}

A simple description for the effective area of SWGO, whose accuracy and validity is sufficient over the energy range of consideration, can be found of the form [4], can be approximately described by a smooth function of the form,

$$
A_{\mathrm{Eff}}(E)=A_{0}\left(\frac{E}{E_{0}}\right)^{4} \prod_{i=1,4}\left(\frac{1}{1+E / E_{i}}\right),
$$

where $A_{0}=100 \mathrm{~m}^{2}, E_{0}=10^{1.4} \mathrm{GeV}, E_{1}=10^{1.7} \mathrm{GeV}, E_{2}=10^{2.1} \mathrm{GeV}, E_{3}=10^{2.5} \mathrm{GeV}$, $E_{4}=10^{3} \mathrm{GeV}$. To obtain the rates of cosmic rays detectable by the instrument, this area is convolved with the cosmic ray spectrum below the knee, motivated by the spectral measurements in [5] of the form

$$
\frac{d \dot{N}}{d E d A}=5 \times 10^{4} E_{\mathrm{GeV}}^{-2.7} e^{\left(-E_{\mathrm{GeV}} / 10^{6} \mathrm{GeV}\right)} \mathrm{m}^{2} \mathrm{~s}^{-1} \mathrm{sr}^{-1} \mathrm{GeV}^{-1}
$$


From this we obtain the expected annual rates of events expected in logarithmic energy bins of size $\Delta E / E$.

$$
E \frac{\Delta N}{\Delta E}=\Delta t_{\mathrm{yr}} \int_{E-\Delta E / 2}^{E+\Delta E / 2} \frac{d \dot{N}}{d E d A} A_{\mathrm{eff}}(E) d E
$$

Using these rates the energy range that can probe a $10^{-3}$ level of anisotropy, assuming a full instrument year's worth of exposure, can then be determined. The left-hand panel of fig. 2 shows the rates obtained, and the right-hand panel indicates the energy range of the anisotropy probe (note that the blue dashed line indicates the cosmic ray anisotropy level intended to be probed).
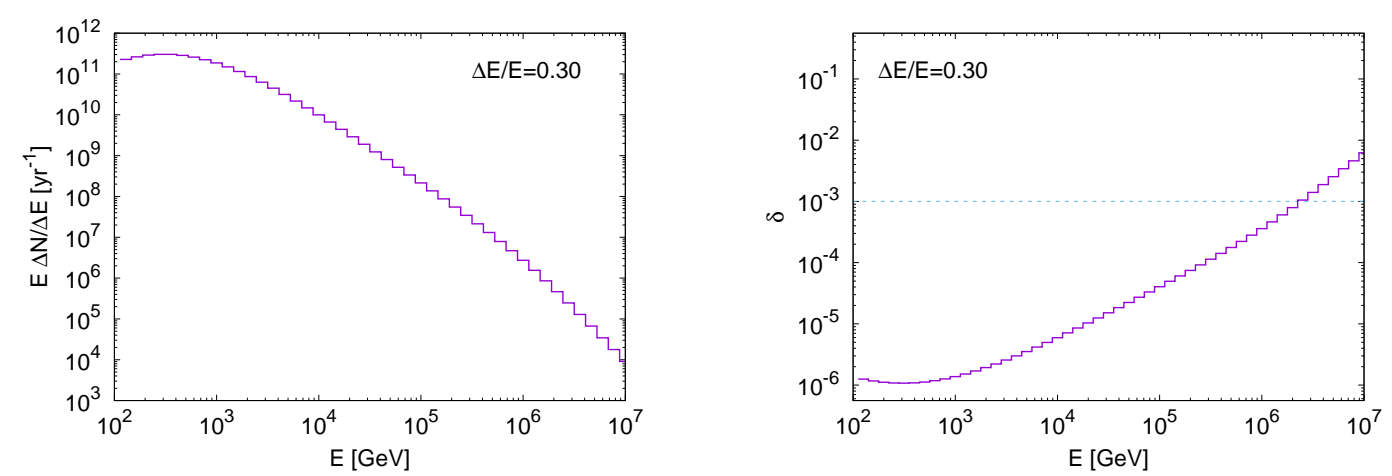

Figure 2: Left-panel: the cosmic ray annual event rates as a function of energy determined for SWGO. Right-panel: the corresponding statistic limited level of anisotropy able to be probed as a function of energy (the energy at which the blue-dashed line and purple line intersect indicates the approximate energy limit that the dipole can be probed up to).

\section{Multipole Range to be Probed}

In order to estimate the noise power spectrum, we use the approximation from [3] that only depends on pixel-by-pixel Poisson noise and gives a flat spectrum $\mathcal{N}_{\ell}=\mathcal{N}$ given by

$$
\mathcal{N} \simeq \frac{1}{4 \pi} \sum_{i} \frac{w_{i}^{2} \Delta \Omega^{2}}{\sum_{\tau} n_{\tau i}},
$$

where $n_{i}$ is the number of events in pixel $i$ and $\Delta \Omega\left(\theta_{\min }, \theta_{\max }\right)=2 \pi\left(\cos \left(\theta_{\min }\right)-\cos \left(\theta_{\max }\right)\right)$ is the solid angle of observed sky.

We can compare the expected angular power spectrum from the simple model in the form

$$
C_{\ell} \propto 1 /(2 \ell+1)(\ell+2)(\ell+1)
$$

introduced by [2], scaled to the all-sky measurement in [1] in order to estimate the highest observable multi-pole moment $\ell$. The corresponding relative uncertainty, assuming a Gaussian distribution of the individual $a_{\ell m}$ 's, is estimated as

$$
\left\langle\Delta C_{\ell}\right\rangle^{2} / C_{\ell}^{2} \sim 2 /(2 \ell+1) / \text { fsky },
$$


where fsky is the fraction of the sky observed. After one year, SWGO should be sensitive to structures larger than $\sim 35^{\circ}(\ell=5)$ at $100 \mathrm{TeV}$ and larger than $\sim 10^{\circ}(\ell=20)$ at $10 \mathrm{TeV}$.

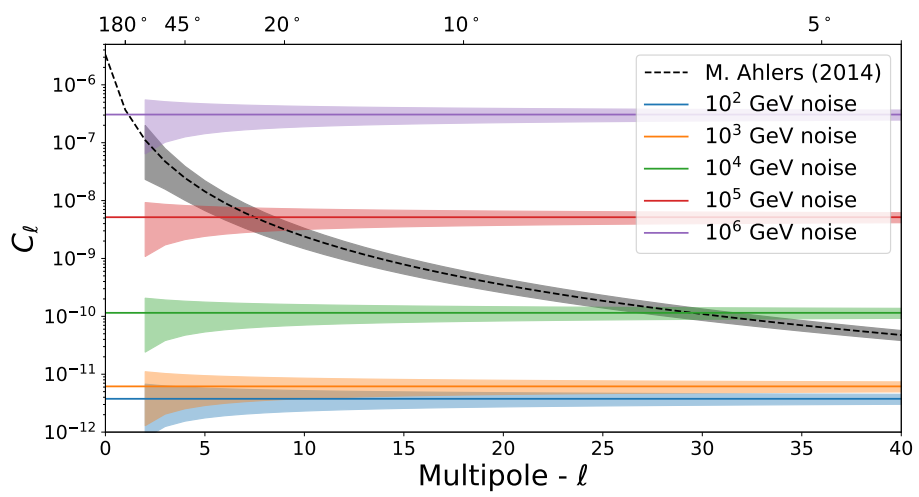

Figure 3: Expected sensitivity to different angular scales for different energies after 1 year. Sensitivity is limited by statistics. The colored solid lines correspond to the angular power spectrum from Poisson noise calculated from Eq. 4 at different energies, and dashed curve shows the model expectation for angular power spectrum from [2].

\section{Separation Power into Mass Groups}

In order to simulate air showers, we use the CORSIKA 7.7400 simulation package [6]. For the standard simulated event set, we select the hadronic interaction model QGSJet-II.04 [7] for energies above $80 \mathrm{GeV}$. UrQMD 1.3.1 [8,9] treats the low energy hadronic interactions. For electromagnetic processes, we use the EGS4 electromagnetic model [10]. Simulations were run for proton, helium, nitrogen and iron primary nuclei species, at an altitude of $4700 \mathrm{~m}$ with a spectral index of -2 for energy range from $10^{3}$ to $10^{6} \mathrm{GeV}$ and Zenith angle from $0^{\circ}$ to $65^{\circ}$. All ground level arriving muons within a radius of $150 \mathrm{~m}$ from the shower core were assumed to be counted by the detector. As indicated from fig. 4, this level of muon counting as a function of the electromagnetic energy deposited on the ground for particles with energy above $10 \mathrm{MeV}$ by the instrument would allow arriving cosmic ray showers to be separated into four distinct mass groups for primary cosmic ray energies above $1 \mathrm{TeV}$.

\section{Conclusion}

The proposed future instrument SWGO offers unique capabilities in terms of its anticipated large effective area, and good muon counting capabilities, and good energy resolution. Utilising these capabilities, our results indicate the great potential that this future instrument offers for cosmic ray anisotropy studies. Specifically, our results indicate that this instrument is well suited for probing the origin of the evolution of the dipole and multipoles of the arriving cosmic rays in the two energy decades below the knee. Beyond sufficient cosmic ray statistics to probe this anisotropy, the separation power between several mass groups offers the potential to shed new light on the underlying physical origin of the anisotropy evolution with energy. 


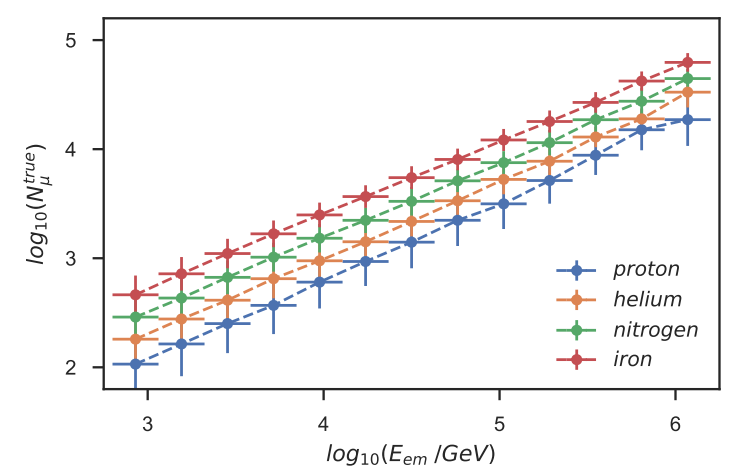

Figure 4: Expected $\mu^{ \pm}$content of mass groups as a function of the electromagnetic energy deposited on the ground for particles with energy above $10 \mathrm{MeV}$ simulated with -2 as slope of the primary energy for showers from $10^{3}$ to $10^{6} \mathrm{GeV}$ and Zenith of from $0^{\circ}$ to $65^{\circ}$ within $150 \mathrm{~m}$ from the shower core.

\section{References}

[1] A. U. Abeysekara et al. [HAWC and IceCube], Astrophys. J. 871 (2019) no.1, 96 doi:10.3847/1538-4357/aaf5cc [arXiv:1812.05682 [astro-ph.HE]].

[2] M. Ahlers, Phys.Rev.Lett. 112 (2014) no. 2, 021101 doi:10.1103/PhysRevLett.112.021101

[3] M. Ahlers et al., Astrophys. J. 823 (2016) no.1, 10 doi:10.3847/0004-637X/823/1/10 [arXiv:1601.07877 [astro-ph.HE]].

[4] A. Albert, R. Alfaro, H. Ashkar, C. Alvarez, J. Alvarez, J. C. Arteaga-Velázquez, H. A. Ayala Solares, R. Arceo, J. A. Bellido and S. BenZvi, et al. [arXiv:1902.08429 [astro-ph.HE]].

[5] W. D. Apel, J. C. Arteaga-Velázquez, K. Bekk, M. Bertaina, J. Blümer, H. Bozdog, I. M. Brancus, E. Cantoni, A. Chiavassa and F. Cossavella, et al. Astropart. Phys. 47 (2013), 54-66 doi:10.1016/j.astropartphys.2013.06.004 [arXiv:1306.6283 [astro-ph.HE]].

[6] Heck, D., et al., CORSIKA: a Monte Carlo code to simulate extensive air showers. (FZKA 6019)(Karlsruhe: Forschungszentrum Karlsruhe) (1998).

[7] S. Ostapchenko, Phys. Rev. D 83, 014018 (2011). https://doi .org/10.1103/PhysRevD. 83.014018

[8] S.A.Bass et al. Prog.Part.Nucl.Phys. 41 (1998) 225

[9] M.Bleicher et al. J.Phys. G25 (1999) 1859

[10] Nelson, W. \& Namito, Yoshihito. (1990). The EGS4 Code System: Solution of gamma-ray and electron transport problems. 